\title{
Potencial de utilização do resíduo de soja como carga em polietileno de baixa densidade (PEBD)
}

\author{
Potential for using soybean wastes as \\ a filler in low-density polyethylene (LDPE)
}

\begin{abstract}
Thalita Paula dos Santos ${ }^{1}$, Hudson Venâncio Silva Garcia ${ }^{1}$, Thaynara Andrade Lopes ${ }^{1}$, Carolina Aparecida dos Santos ${ }^{1}$, Ana Carolina Corrêa Furtini', Lourival Marin Mendes ${ }^{1}$, José Benedito Guimarães Júnior ${ }^{1}$
\end{abstract}

\footnotetext{
${ }^{1}$ Universidade Federal de Lavras, Programa de Pós-graduação em Engenharia de Biomateriais, CP: 3037, CEP: 37200 900, Lavras, Minas Gerais, Brasil.

e-mail: thalitasantospp@hotmail.com, hudsonjapa@hotmail.com, thaynaraandradezps@gmail.com, carolinaapnep@gmail.com, carol.furtini@gmail.com, lourival@dcf.ufla.br, jose.guimaraes@ufla.br
}

\section{RESUMO}

O descarte inadequado de polímeros de origem petrolífera contribui para o aumento da poluição ambiental devido ao grande período de tempo que estes materiais levam para degradar. Assim, o desenvolvimento de materiais poliméricos reforçados com resíduos de materiais lignocelulósicos pode proporcionar uma melhor taxa de biodegradação ao polímero, por se tratar de materiais de origem natural, renovável e biodegradável. Neste sentido, o objetivo desta pesquisa foi verificar o potencial de utilização do resíduo de soja como carga em polietileno de baixa densidade (PEBD) através de caracterização físico-mecânica. Foram avaliadas diferentes composições do resíduo de soja em relação à massa total de PEBD $0 \%, 5 \%, 10 \%, 15 \%$ e $20 \%$. O resíduo de soja utilizado foi caracterizado quimicamente para determinação do teor de extrativos, lignina, cinzas e hemicelulose. Os pellets formados por extrusão foram prensados a uma temperatura de $160^{\circ} \mathrm{C}$ sem pressão durante 12 minutos, onde se obteve os corpos de prova para os ensaios físico-mecânicos. Após a produção, os mesmos foram acondicionados em sala de climatização com temperatura de $20 \pm 2^{\circ} \mathrm{C}$ e umidade relativa de $65 \pm 3 \%$, sendo posteriormente realizada a determinação da densidade aparente, umidade na base seca, absorção total de água e realizados de flexão e tração. Com base nos resultados, conclui-se que os valores encontrados para os constituintes químicos do resíduo de soja mostram satisfatórios, uma vez que, a substituição parcial do PEBD puro pelo não afetou as propriedades de densidade e resistência à tração do material. O aumento do teor deste resíduo, também contribui para um maior alongamento do polímero. Assim, conclui-se que é possível transformar este polímero de origem petrolífera em um material mais sustentável, tornando algumas propriedades até mais atrativas do que aquelas encontradas no PEBD puro.

Palavras-chave: Materiais poliméricos. Resíduos lignocelulósicos. Sustentabilidade.

\section{ABSTRACT}

The improper disposal of polymers of petroleum origin contributes to the increase of environmental pollution due to the long period of time that these materials take to degrade. Thus, the development of polymeric materials reinforced with residues of lignocellulosic materials can provide a better rate of biodegradation to the polymer, as they are materials of natural, renewable and biodegradable origin. In this sense, the objective of this research was to verify the potential of using soybean residue as a filler in low density polyethylene (LDPE) through physical-mechanical characterization. Different soy residue compositions were evaluated in relation to the total mass of LDPE $0 \%, 5 \%, 10 \%, 15 \%$ and $20 \%$. The soybean residue used was chemically characterized to determine the content of extractives, lignin, ash and hemicellulose. The pellets formed by extrusion were pressed at a temperature of $160^{\circ} \mathrm{C}$ without pressure for 12 minutes, where the specimens were obtained for the physical-mechanical tests. After production, they were stored in an air conditioning room with a temperature of $20 \pm 2{ }^{\circ} \mathrm{C}$ and a relative humidity of $65 \pm 3 \%$, with the determination of apparent 
density, moisture on the dry basis, total water absorption and subsequently carried out. flexion and traction. Based on the results, it is concluded that the values found for the chemical constituents of the soy residue are satisfactory, since the partial substitution of pure LDPE by the material did not affect the density and tensile strength properties of the material. The increase in the content of this residue also contributes to a greater elongation of the polymer. Thus, it is concluded that it is possible to transform this petroleum-based polymer into a more sustainable material, making some properties even more attractive than those found in pure LDPE..

Keywords: Polymeric materials. Lignocellulosic waste. Sustainability.

\section{INTRODUÇÃO}

O desenvolvimento de materiais sustentáveis tornou um parâmetro importante para civilização humana nos dias de hoje. A adição de materiais residuais em materiais poliméricos, reduzem os problemas de descarte associados a eles, uma vez que são biodegradáveis, renováveis e podem apresentar satisfatória relação resistência/peso, podendo, no entanto, oferecer propriedades superiores em relação aos materiais convencionais [1].

A aplicação das fibras vegetais como reforços têm atraído o interesse de pesquisadores já que podem fornecer propriedades desejáveis de maneira sustentável e a um custo acessível. Atualmente, vários polímeros sintéticos vêm sendo utilizados como matrizes para fabricar compósitos, que podem ser aplicados nos setores automotivos, eletrônico, civil, de embalagens e biomédico [2].

O polietileno de baixa densidade (PEBD), por exemplo, é amplamente utilizado em diversas aplicações industriais, apesar de suas diversas aplicações, existem preocupações crescentes em relação à geração de resíduos e à natureza não biodegradável $[3,4]$ Em relação às suas aplicações, podem ser utilizados como filmes na fabricação de embalagens alimentícias, industriais, farmacêuticas e agrícolas, assim como, em brinquedos, revestimentos de fios e cabos, utilidades domésticas, tubos e mangueiras [5].

Esforços significativos vêm sendo feitos para desenvolver materiais biodegradáveis à base de PEBD com adição de fibras vegetais, uma vez que aumentaria a taxa de biodegradação e reduziria a utilização global do PEBD e a dependência mundial de recursos petrolíferos não renováveis [6]. A aplicação de fibras vegetais como reforço em materiais poliméricos, seria uma opção, pois estas são encontradas em abundância na natureza, são de origem renovável, são biodegradáveis e relativamente baratas quando comparadas aos reforços sintéticos convencionais, como as fibras de vidro, por exemplo [4].

A soja, por exemplo, é um dos principais produtos agrícolas no mundo, sendo o Brasil o segundo maior produtor mundial de soja em grãos [7]. No Brasil estima-se uma produção de 135,5 milhões de toneladas de soja para a safra 2020/2021 [8]. Sendo que para a produção total obtida, cerca de $39 \%$ são grãos e o restante palhada. Da secagem do grão de soja, resulta o resíduo de limpeza de soja, que pode ser utilizado na alimentação de bovinos, como uma fonte proteica de baixo custo. Porém, a grande variabilidade na composição bromatológica de resíduos dificulta o balanceamento nutricional das dietas, se tornando um problema de uso para essa finalidade [9].

Neste sentido, este trabalho objetivou produzir e analisar as propriedades físicas e mecânicas do polietileno de baixa densidade com cargas de resíduo de soja, buscando desenvolver um material sustentável, mais leve, de menor custo e que possua propriedades semelhantes ou superiores àquelas encontradas no PEBD puro.

\section{MATERIAIS E MÉTODOS}

As cascas de soja foram adquiridas em uma empresa que atua no mercado de commodities e operações, na Unidade de Jataí, no sudoeste de Goiás. O resíduo utilizado foi beneficiado (separação casca e semente), sendo posteriormente peneirado e lavado. Para análise química do resíduo utilizado, as partículas foram moídas em um moinho tipo Willey e peneiradas, sendo utilizadas as que ficaram retidas entre as peneiras de 40 mesh $(0,420 \mathrm{~mm})$ e 60 mesh $(0,250 \mathrm{~mm})$. Posteriormente, as partículas do resíduo de soja foram levadas à estufa durante 24 horas a uma temperatura de $105^{\circ} \mathrm{C}$ para secagem completa. A determinação dos extrativos totais foi realizada com tolueno-etanol e etanol, seguindo a metodologia descrita na NBR 14853 [10]. Para lignina insolúvel, o H2SO4 foi utilizado conforme descrito na NBR 7989 [11], o teor de cinzas foi determinado de acordo com a NBR 13999 [12] utilizando um forno tipo mufla. Já o teor de holocelulose foi determinado pela diferença, conforme a equação: 
O polietileno de baixa densidade (PEBD) foi adquirido da Proex Embalagens Plásticas, localizada em Lavras, Estado de Minas Gerais, Brasil. O material tinha dimensões de aproximadamente 2 × $4 \mathrm{~cm}$, no qual foi manualmente misturado ao resíduo de soja em cinco composições diferentes (Tabela 1) para ser fabricado o material polimérico com resíduo de soja pelo processo de extrusão. Foi utilizada uma extrusora dupla-rosca co-rotante modelo SJSL-20, instalada no Departamento de Engenharia da Universidade Federal de Lavras, na qual a mistura é depositada em um funil de alimentação que a transfere para a região interna da extrusora, envolta por um cilindro aquecido, onde as roscas são responsáveis por misturar, fundir, plastificar e transportar o material até a matriz final que modela o material em dois filamentos que são imersos em água para que ocorra seu resfriamento. O perfil de temperatura foi mantido em todas as composições, passando por seis zonas cujas temperaturas aumentaram gradualmente conforme a seguinte sequência: $110^{\circ} \mathrm{C}-120^{\circ} \mathrm{C}-120^{\circ} \mathrm{C}$ $-130{ }^{\circ} \mathrm{C}-130{ }^{\circ} \mathrm{C}-140{ }^{\circ} \mathrm{C}$ e a velocidade da rosca foi de $80 \mathrm{rpm}$. Por fim, estes filamentos são inseridos em uma peletizadora que os transforma em pellets (Figura 1).

Tabela 1: Composições dos materiais fabricados.

\begin{tabular}{c|c|c}
\hline COMPOSIÇÃo & RESíDUO DE SOJA (\%) & PEBD (\%) \\
\hline 1 & 0 & 100 \\
\hline 2 & 5 & 95 \\
\hline 3 & 10 & 90 \\
\hline 4 & 15 & 85 \\
\hline 5 & 20 & 80 \\
\hline
\end{tabular}
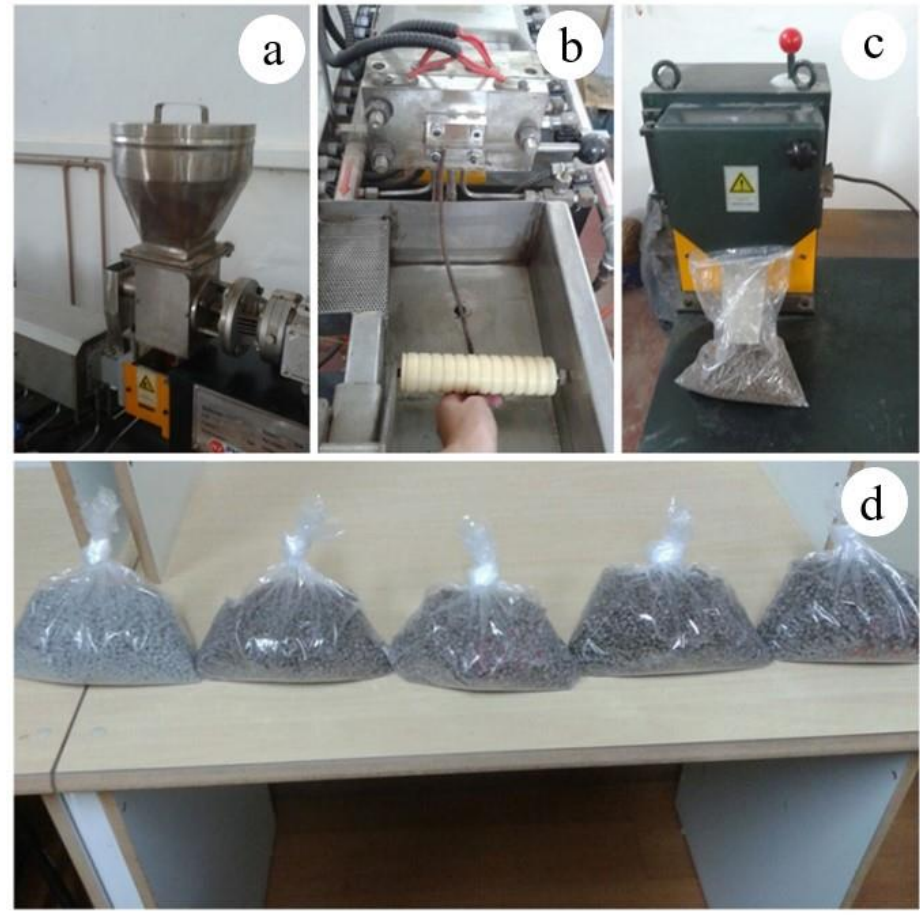

Figura 1: Funil de alimentação da extrusora (a), material conformado em forma de fio sendo resfriado por água na extrusora (b), formação de pellets (c), materiais pelletizados separados por diferentes composições (d).

Os pellets formados pelo processo de extrusão foram cortados em pequenos grânulos, do qual foram colocados em um molde metálico com as dimensões de 12 × 1,5 x 0,3 cm (largura, comprimento e espessura) e dispostos para preencher a área para evitar a formação de espaços vazios e encaminhados para a prensa hidráulica Marcon 15 toneladas com temperatura de $160^{\circ} \mathrm{C}$ sem adição de pressão durante 12 minutos, sendo confeccionado os corpos de prova sendo obtido 5 lâminas de cada composição do material laminado. 
Após a fabricação, as lâminas foram acondicionadas em sala de climatização com temperatura de $20 \pm 2{ }^{\circ} \mathrm{C}$ e umidade relativa de $65 \pm 3 \%$. Posteriormente, foram retirados corpos de provas para avaliação de suas propriedades físicas e mecânicas. Os ensaios realizados foram flexão estática MOE e MOR, conforme a norma ASTM-D790-03 [13], tração, de acordo com a norma ASTM-D638 [14] no qual foi realizado pela máquina universal EMIC DL-30000, localizada na Unidade Experimental de Painéis de Madeira (UEPAM) da UFLA, para a absorção de água após 24h de imersão e teor de umidade, conforme a ASTM -D570 [15], já a densidade aparente foi determinada conforme a equação:

$$
\rho=M / V
$$

$$
\begin{aligned}
& \text { Onde: } \\
& \rho=\text { Densidade Aparente }\left(\mathrm{g} / \mathrm{cm}^{3}\right) \\
& \mathrm{M}=\text { Massa do corpo de prova }(\mathrm{g}) ; \\
& \mathrm{V}=\text { Volume do corpo de prova }\left(\mathrm{cm}^{3}\right)
\end{aligned}
$$

Os resultados obtidos nas caracterizações realizadas foram avaliados no programa SISVAR, utilizando o teste estatístico Scott Knott a 5\% de significância.

\section{RESULTADOS E DISCUSSÃO}

\subsection{Caracterização Química}

São apresentados na Tabela 2 os resultados adquiridos da análise química do resíduo de soja.

Tabela 2: Resultados médios da análise química do resíduo de soja.

\begin{tabular}{l|c}
\hline \multicolumn{1}{c|}{ ANÁLISE QUÍMICA } & TEOR (\%) \\
\hline Umidade (\%) & $3,01 \pm 0,80$ \\
\hline Extrativos totais (\%) & $19,95 \pm 3,18$ \\
\hline Lignina solúvel (\%) & $37,83 \pm 2,12$ \\
\hline Cinzas (\%) & $7,37 \pm 0,49$ \\
\hline Holocelulose (\%) & $34,85 \pm 2,03$ \\
\hline
\end{tabular}

O teor de umidade encontrado neste estudo se mostra inferior ao adquirido por Silva et al. [16] que estudaram o resíduo de soja e obtiveram um valor médio de $8,40 \%$. O valor de 3,01\% foi considerado um teor satisfatório, pois um alto teor de umidade pode provocar uma rede de microfraturas na superfície dos PEBD com resíduos de soja alterando suas propriedades físicas e mecânicas, resultando assim no aumento da dimensão do material e prejudicando aplicação em trabalhos de precisão [17]. Quanto maior a umidade, maior o volume de gases gerados, no interior, cabeçote e matriz da extrusora, com formação de bolhas, manchas superficiais e dificuldade da adesão matriz/resíduo durante o processo [18,19].

Para os valores de extrativos totais, Martins et al. [20] encontraram um teor superior de 26,72\% para o resíduo de soja aplicado na produção de painel aglomerado. Conforme Shebani et al. [21] polietileno de baixa densidade com materiais lignocelulósicos de baixos teores de extrativos, pode proporcionar um aumento de estabilidade térmica para um compósito. Já Sheshmani et al. [21] indicam que baixos teores de extrativos em materiais termoplásticos, pode proporcionar um aumento nas propriedades mecânicas. Logo, o valor encontrado no presente estudo mostra um valor satisfatório.

O teor de lignina encontrado neste estudo pode ser um fator benéfico caso ocorra um adequado acoplamento entre a matriz PEBD e a fase dispersa das fibras dos resíduos de soja, uma vez que, que fibras vegetais com alto teor de lignina podem apresentar maior rigidez, maior resistência à compressão e menor absorção de água, tendo em vista que, segundo Bathia et al. [22], a lignina pode conferir impermeabilidade, rigidez e resistência a ataques microbiológicos e mecânicos aos tecidos vegetais. Comparado ao estudo realizado por LOPES et al. [23] que ao estudarem o resíduo de soja, identificaram um teor de $23 \%$, valor inferior ao en- 
contrado neste estudo.

Conforme Barrichelo e Brito [24], altos teores de cinzas podem ser um fator prejudicial e, em alguns casos, limitante ao uso de matérias-primas lignocelulósicas, pois elevados teores podem prover desgaste excessivo em equipamentos cortantes, tais como moinhos e serras. No estudo de Silva et al. [16], foi encontrado um valor semelhante ao teor de cinzas para o resíduo da soja, os quais observaram valor médio de 8,90\%, mostrando assim que o teor encontrado no presente estudo está de acordo com a faixa normalmente relatada em literatura.

O teor de holocelulose é importante devido sua lenta degradação térmica, portanto os produtos obtidos a partir da decomposição destes componentes vão obter um rendimento maior [25]. Logo, o valor encontrado mostra satisfatório, uma vez que na literatura os valores demonstram maiores, como Santana et al. [26] que obtiveram um teor de $75,34 \%$.

\subsection{Caracterização Física}

As Figuras 2 e 3 mostram os resultados obtidos para a análise de umidade nas lâminas e densidade, respectivamente.

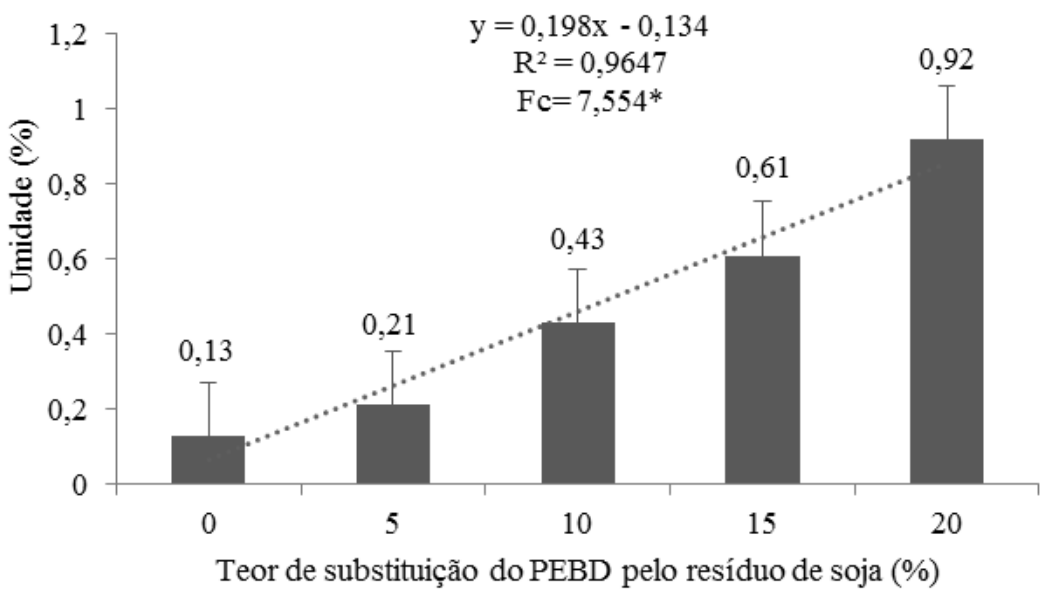

Figura 2: Valores obtidos para umidade das lâminas de acordo com a porcentagem de resíduo de soja.

* Significativo (valor de $\mathrm{p}<0,05$ ); $\mathrm{y}=$ Umidade; $\mathrm{R}^{2}=$ Coeficiente de determinação e $\mathrm{Fc}=$ Fator de correção

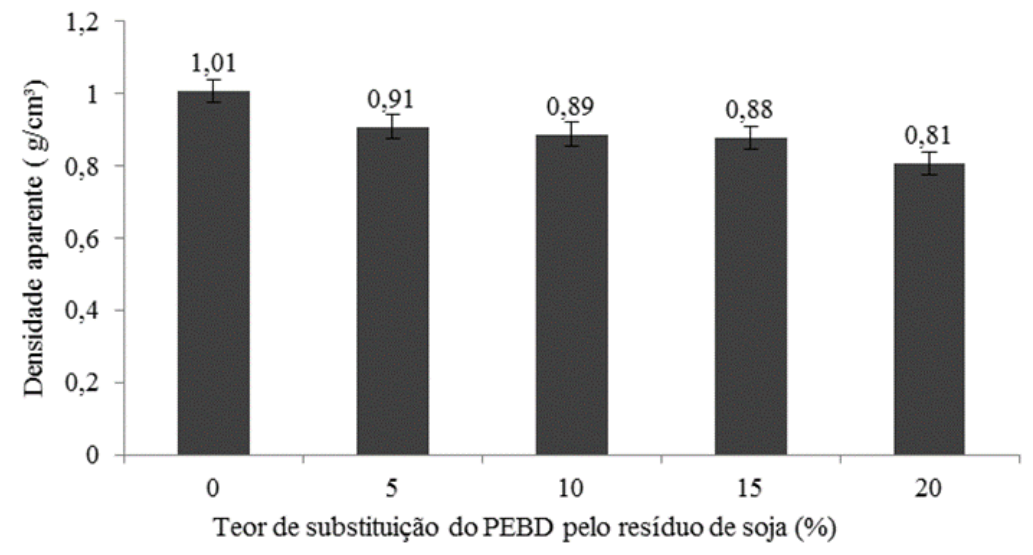

Figura 3: Valores obtidos para densidade aparente das lâminas, de acordo com a porcentagem de resíduo de soja.

Observa-se na Figura 2 que ao acrescentar 1\% de resíduo houve um aumento de 0,19\% de umidade. Essa umidade trata do equilíbrio entre a umidade relativa e a temperatura do ar ambiente podendo ser influenciada pelos processos aplicados, como prensagem e temperatura, e ainda pelos constituintes do material. O teor de umidade do PEBD com resíduo de soja sofreu um acréscimo significativo em relação ao PEBD puro, esse fato pode ser explicado devido à composição química do resíduo de soja. Como a holocelulose é um dos principais componentes químicos presente nos resíduos de soja Qing et al [27], esse componente atribui um 
caráter higroscópico ao resíduo Chinta et al [28]. Logo, os materiais com maiores teores de resíduos, apresentaram maiores teores de umidade, diferente dos polímeros que são materiais hidrofóbicos.

De acordo com a Figura 3, observa-se a diminuição da densidade aparente nos PEBD com o acréscimo do resíduo. Essa redução indica uma vantagem, pois reduz os gastos com as manutenções dos equipamentos utilizados no processamento, devido os menores desgastes de ferramentas e as baixas temperaturas de processamento Poletto [29].

Na Figura 4 são exibidos os valores obtidos para as propriedades de absorção de água (AA) em 24 horas de imersão.

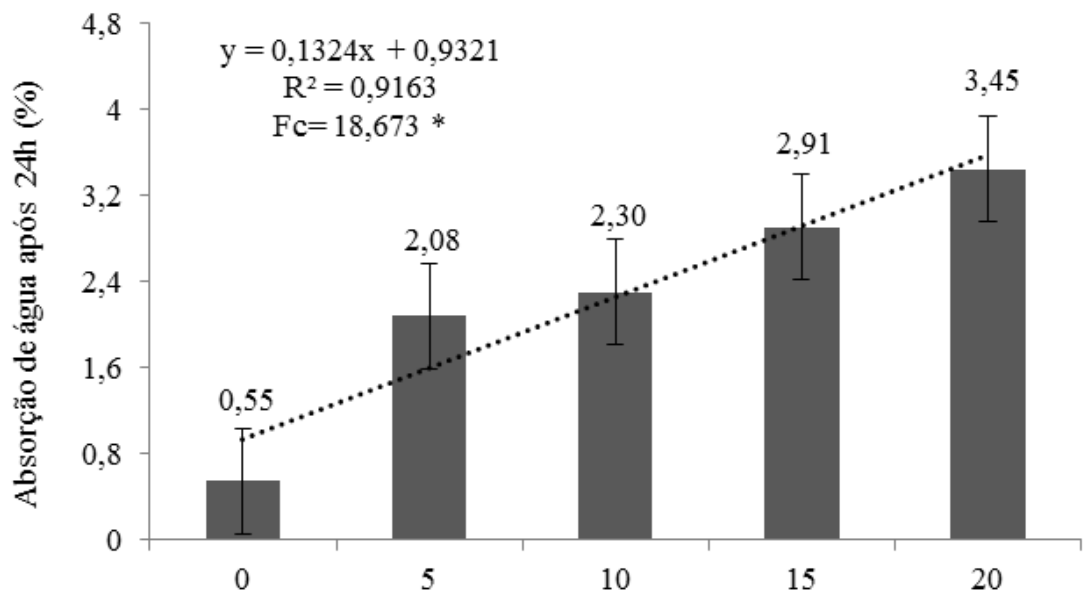

Teor de substituição do PEBD pelo resíduo de soja (\%)

Figura 4: Variação de absorção de água (AA) em 24 horas de imersão, de acordo com a porcentagem de resíduo de soja. * Significativo (valor de $\mathrm{p}<0,05$ ); $\mathrm{y}=$ Absorção de água; $\mathrm{R}^{2}=$ Coeficiente de determinação e Fc= Fator de correção

O acréscimo de $1 \%$ na quantidade de resíduo de soja inserido no PEBD provocou aumento nos valores de absorção de água de $0,13 \%$. Tal fato pode ser explicado pelos diferentes tipos de resíduos usados na fabricação, o que influencia na capacidade de absorção de água, que é específica à cada fibra vegetal.

O índice de absorção de água pelo PEBD com resíduo de soja é considerado baixo mesmo quando comparado a materiais poliméricos com reforço de materiais lignocelulósicos. Materiais poliméricos de composição similar contendo resíduo de madeira em pó, foram estudados por Teixeira et al. [30] encontrando valores de absorção de água variando entre $1,44 \%$ e $3,77 \%$. Outros estudos como o de Tita et al. [31], introduziram as fibras vegetais em compósitos fenólicos elevando o percentual de absorção de água em comparação com o termofixo puro. Este resultado foi semelhante ao obtido neste trabalho, em que o PEBD puro exibiu um menor valor em comparação as demais composições.

\subsection{Caracterização Mecânica}

As Figuras 5 e 6 apresentam os resultados médios obtidos para o módulo de elasticidade (MOE) e módulo de ruptura (MOR) no ensaio de flexão estática. 


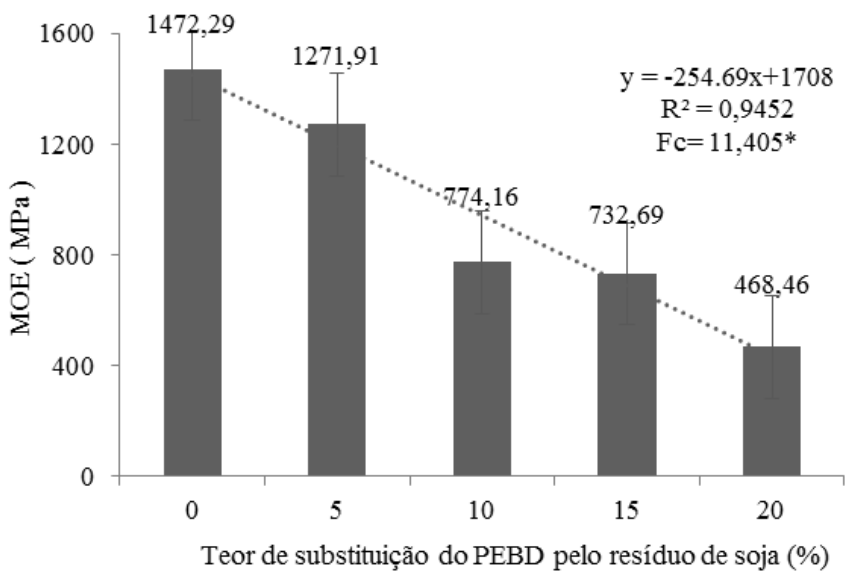

Figura 5: Valores do módulo de elasticidade (MOE) em função da porcentagem utilizada do PEBD com resíduo de soja. * Significativo (valor de $\mathrm{p}<0,05$ ); $\mathrm{y}=$ MOE $\mathrm{R}^{2}=$ Coeficiente de determinação e $\mathrm{Fc}=$ Fator de correção

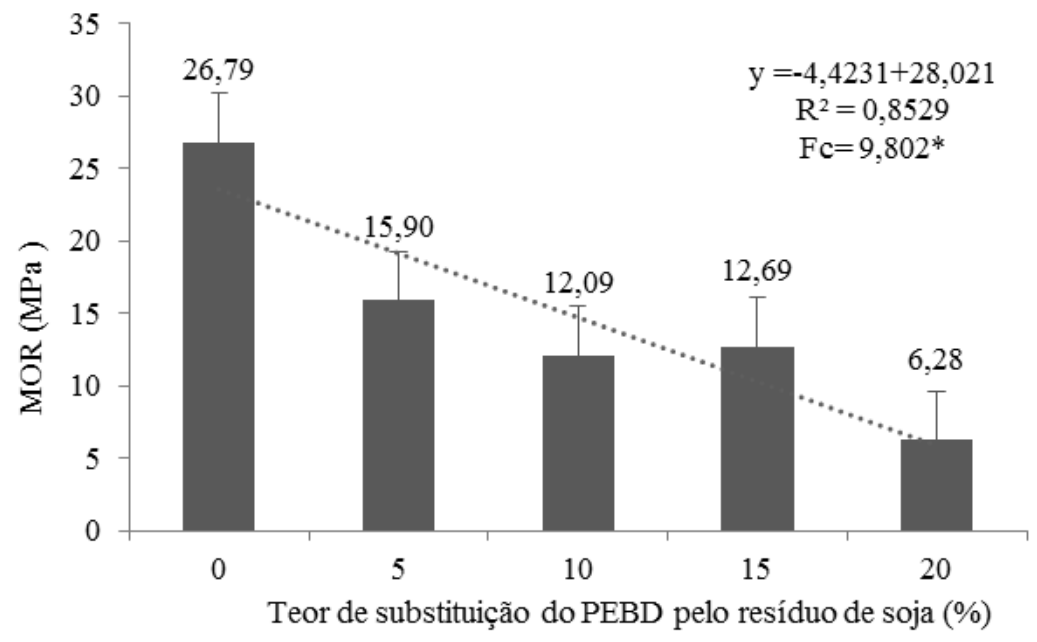

Figura 6: Valores do módulo de ruptura (MOR) em função da porcentagem utilizada do PEBD com resíduo de soja. * Significativo (valor de $\mathrm{p}<0,05$ ); $\mathrm{y}=\mathrm{MOR} ; \mathrm{R}^{2}=$ Coeficiente de determinação e $\mathrm{Fc}=$ Fator de correção

Houve efeito significativo com o acréscimo de reforço de partículas do resíduo sobre as propriedades módulo de elasticidade (MOE) e módulo de ruptura (MOR) à flexão estática. Ao acrescentar 1\% de resíduo houve um decréscimo de 254,69 MPa para o MOE e 4,42 MPa ao MOR. Essa redução nos módulos de elasticidade e ruptura pode ter ocorrido devido à presença de vazios que atuaram como agentes de falha e levaram à quebra do material quando submetido à tensão. Adicionalmente, o aumento de fibras pode levar a área com regiões com acúmulo do PEBD e área com concentração de fibra, dificultando o contato do polímero com a fibra e produzindo a redução da adesão [32]. Supõe-se que a incompatibilidade desses componentes, pode ter sido responsável pela baixa aderência, o que causou a diminuição das propriedades [33]; logo, um agente de acoplamento ou a modificação da superfície das fibras seria necessário para aumentar essa adesão [34].

Guedes et al. [35] ao trabalharem com fibras da palmeira real australiana substituindo parcialmente a matriz de PEBD nos teores de 5, 10, 15 e 20\%, também obtiveram uma redução significativa dos resultados médios obtidos para o MOE na flexão. Já para o MOR, estes autores observaram um aumento significativo do mesmo conforme se aumentou o teor das fibras da palmeira, o que mostrou que este resíduo lignocelulósico se comportou diferente da soja para esta propriedade.

As Figuras 7 e 8 exibe os valores médios obtidos para a resistência à tração e tensão deformação respectivamente, do PEBD com resíduo de soja. 


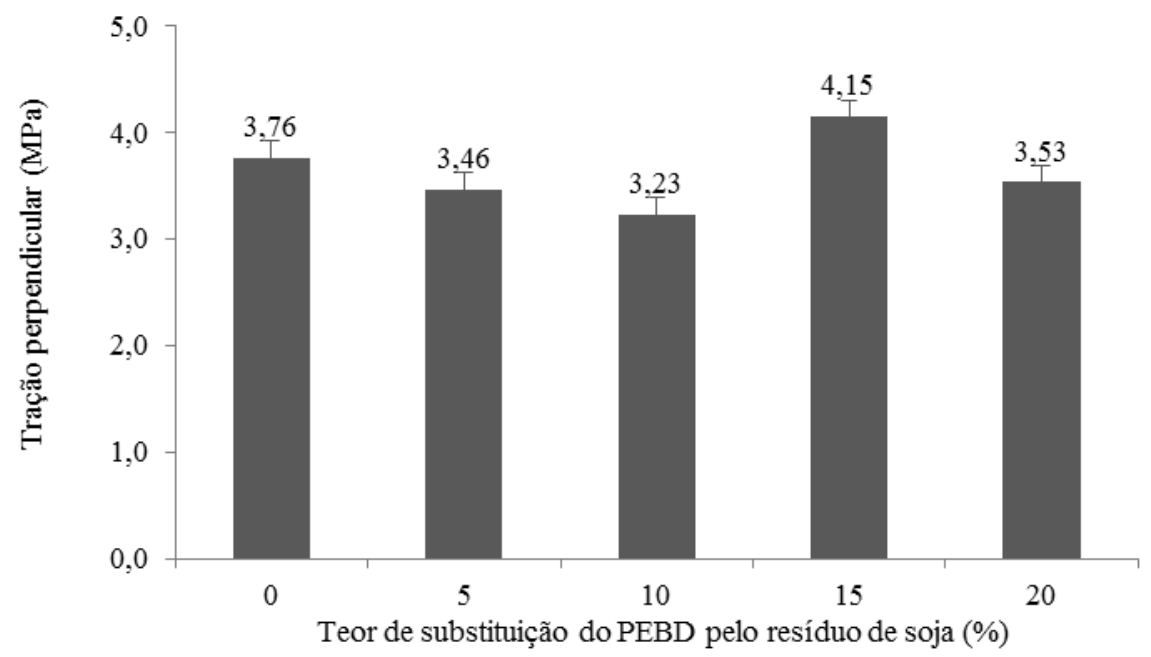

Figura 7: Valores médios obtidos para resistência à tração para o PEBD com resíduo de soja.

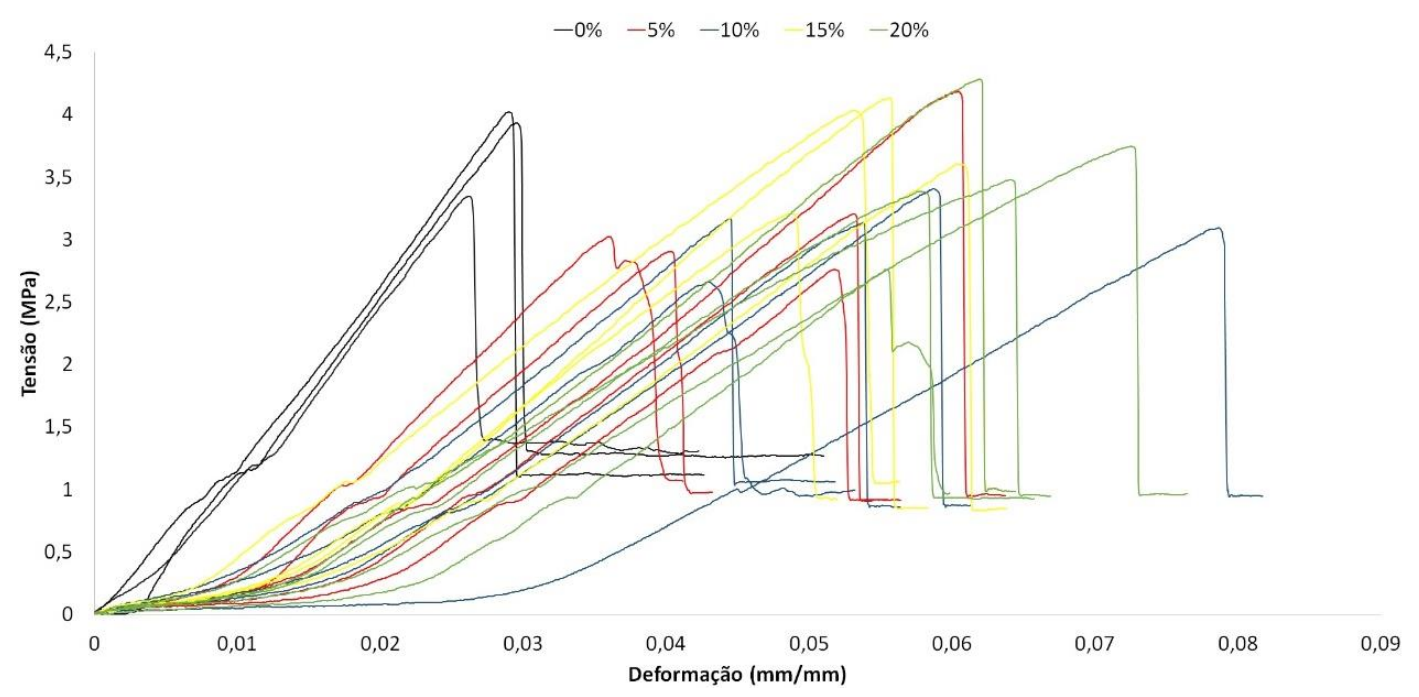

Figura 8: Curvas de Tensão x Deformação do para o PEBD com resíduo de soja.

Para a resistência à tração Figura 7, não houve diferença significativa com o acréscimo do resíduo de soja conforme a porcentagem de fibras adicionadas. Stork e Rocha [36] ao avaliarem a resistência à tração em no PEBD com reforço de torta de mamona nos teores de $0 \%, 5 \%, 10 \%, 20 \%$ e $30 \%$, observaram a mesma tendência, ou seja, não houve diferença significativa à medida que o teor de reforço do material lignocelulósico foi aumentado. Esta variação não significativa dos resultados pode ser atribuída ao fato de que o PEBD adquiriu parte da rigidez das fibras do material lignocelulósico, o que impediu que este passasse por grandes deformações [32].

Já Wearn et al estudando o PEBD com fibras de coco tratadas quimicamente por extração alcalina de hidróxido de sódio $(\mathrm{NaOH})$, relataram que o aumento do teor de fibra de coco em massa reduziu ligeiramente a resistência à tração do compósitos. Do mesmo modo Veloso et al, que substituíram o PEBD por biomassa de cacau e obtiveram uma média de 4,98MPa para a substituição de $20 \%$.

Na figura 8, apresenta as curvas Tensão x Deformação para o PEBD com a inserção do resíduo de soja, de forma a ilustrar o comportamento mecânico do material. Contudo, observou-se que ao aumentar o teor de fibras na matriz de PEBD houve uma maior deformação do material, fato este que no estudo de Rocha e Mulinari [37] associam a um aumento da ductibilidade do material, devido ao reforço utilizado.

Fato semelhante ao estudo de Moreira e Seo [38] observou-se que a adição de fibras no polímero aumentou a deformação do material, e deformação plástica. Eesse fenômeno pode ser explicado pois em um 
processamento por extrusão, as propriedades do polímero e do reforço podem mudar, pois o esforço mecânico é aplicado ao mesmo que o calor sobre o polímero fundido

A Figura 9 apresenta os resultados obtidos para o alongamento específico dos do PEBD com resíduo de soja na tração.

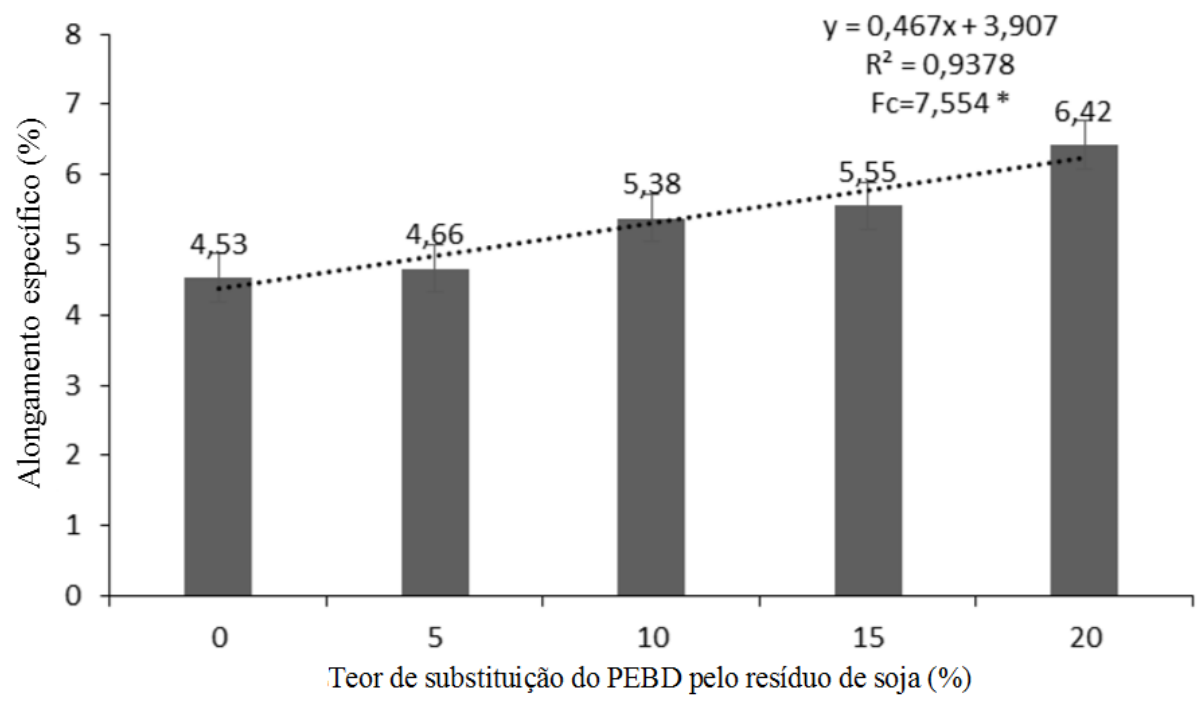

Figura 9: Valores médios obtidos para o alongamento específico para o PEBD com resíduo de soja.

* Significativo (valor de $\mathrm{p}<0,05$ ); $\mathrm{y}=$ Alongamento específico; $\mathrm{R}^{2}=$ Coeficiente de determinação e $\mathrm{Fc}=$ Fator de correção

Observa-se que ao acrescentar $1 \%$ do resíduo de soja, o alongamento específico aumentou 0,46\%. Este comportamento indica uma redução da rigidez da matriz com o acréscimo do teor de soja. Comparando o PEBD puro, apenas a composição com acréscimo de 5\% de soja foi estatisticamente igual. Essas duas composições diferiram estatisticamente das demais, sendo que as composições com $10 \%$ e $15 \%$ de teor de soja também são estatisticamente iguais entre si. O resultado encontrado para o alongamento específico para o PEBD puro é próximo ao encontrado por Oliveira et al. [39] que obtiveram 5,6 \% para essa matriz polimérica pura e 4,4 \% para a matriz com adição de 5\% de fibra de coco verde. Embora os resultados sejam próximos, observou-se que o acréscimo de soja gerou efeito de aumento no alongamento da matriz, enquanto as fibras de coco verde estudada pelos autores citados geram um comportamento inverso.

A Figura 10 exibe os resultados obtidos através do ensaio de tração para o módulo de Young do PEBD com resíduo de soja.

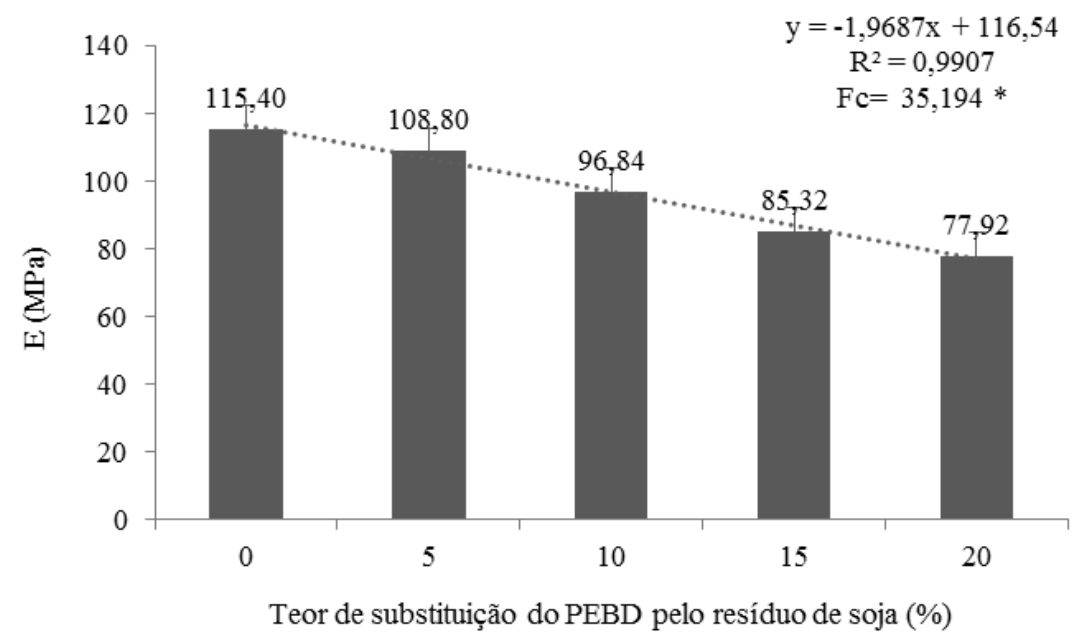

Figura 10: Valores médios obtidos para o módulo de Young para o PEBD com resíduo de soja. * Significativo (valor de p <0,05); $\mathrm{y}=\mathrm{E} ; \mathrm{R}^{2}=$ Coeficiente de determinação e Fc= Fator de correção 
O módulo de elasticidade na tração, também conhecido como módulo de Young, variou significativamente conforme o teor de soja foi aumentado nos materiais, sendo que o aumento de $1 \%$ de soja no PEBD ocasionou em um decréscimo de 1,9687 MPa. Rocha e Mulinari [37] encontraram 52,15 MPa para o PEBD puro, sendo que este valor aumentou com adição de fibras de palmeira de maneira não significativa até a adição de $15 \%$ de fibra vegetal, com os valores de 69,3, 61 e 68,9 MPa para adição de 5\%,10\% e 15\%, respectivamente, chegando a variação de 300,3 MPa para adição de $20 \%$ de fibras da palmeira.

Já Stork e Rocha [36], ao estudarem essa propriedade do PEBD com reforço de torta de mamona encontraram aproximadamente $103 \mathrm{MPa}$ para o PEBD puro, valor próximo ao encontrado neste estudo, seguido por um acréscimo com o aumento do teor de torta de mamona em 5\%, 10\% e 15\%. Na adição de $20 \%$ da torta de mamona houve uma pequena redução desta propriedade, seguido pelo aumento a $30 \%$ de adição.

A redução do módulo de elasticidade para a tração neste trabalho, pode ter ocorrido devido a uma rigidez inferior das fibras em relação à rigidez da matriz de PEBD, o que gera também um aumento do alongamento[40]. Outro fator que pode ter influenciado essa redução, foi a adesão interfacial entre fibra e matriz, logo o acréscimo de um acoplamento ou a modificações das fibras, proporcionaria uma melhor adesão e consequentemente o aumento do módulo de elasticidade

\section{CONCLUSÕES}

Este estudo buscou desenvolver um novo material com o resíduo de soja incorporado ao PEBD, visando uma destinação alternativa a este resíduo do agronegócio, além de uma redução de massa do material polimérico que, muitas vezes é descartado no meio ambiente, levando centenas de anos para se decompor.

O teor de resíduo de soja não afetou significativamente a densidade dos materiais em relação ao PEBD puro, o que é benéfico para aplicações que exijam manter a leveza deste material. O teor de umidade e absorção de água aumentou significativamente com o acréscimo de soja incorporada, devido à natureza higroscópica dos materiais lignocelulósicos. A falta de agentes compatibilizantes pode ter afetado a adesão do PEBD com o resíduo de soja e o que contribuiu para a redução das propriedades de MOE e MOR na flexão, assim como o módulo de Young na tração. Em contrapartida, a resistência à tração não foi afetada com o acréscimo de soja ao PEBD nas diferentes concentrações estabelecidas e para o alogamento houve um aumento com o acréscimo do resíduo.

Esses resultados mostraram que a substituição parcial do PEBD pelos resíduos da soja nos teores estudados é uma opção de substituição ao PEBD puro em aplicações em que são necessárias uma boa resistência à tração e uma melhoria no alongamento do material.

Assim, conclui-se que foi possível transformar este polímero de origem petrolífera em um material mais sustentável, tornando algumas propriedades até mais atrativas do que aquelas encontradas no PEBD puro.

\section{AGRADECIMENTOS}

Os autores agradecem à CAPES, à FAPEMIG e ao CNPq pelo amparo financeiro para realização da pesquisa.

\section{BIBLIOGRAFIA}

[1] DUBEY, S.D., MISHRA, V., SHARMA, A. "A review on polymer composite with waste material as reinforcement." Materials Today: Proceedings, 2021.

[2] BALLA, V.K., KATE, K.H., SATYAYOLU, J., et al., "Additive manufacturing of natural fiber reinforced polymer composites: Processing and prospects", Composites Part B: Engineering, v.174, pp. 1-29, May. 2019.

[3] GEYER, R., JAMBECK, J.R., LAW, K.L. Production, use and fate of all plastics ever made. Science Advances, v.3, n.7, 2017.

[4] MONTAZER, Z., HABIBI NAJAFI, M.B., LEVIN, D.B. Challenges with verifying microbial degradation of polyethylene.Polymers, v. 12, n. 1, pp. 123, 2020. 
[5] COUTINHO, F.M.B., MELlO, I.L., SANTA MARIA, L.C., "Polietilenos: Principais Tipos, Propriedades e Aplicações", Polímeros: Ciência e Tecnologia, v. 13, n. 1, pp. 1-13, 2003.

[6] KABOORANI, A., HAMZEH,Y., ABDULKHANI,A., et al., Tailoring the low-density polyethylene thermoplastic starch composites using cellulose nanocrystals and compatibilizer. Polymer Testing, v. 93, 2021.

[7] COMPANHIA NACIONAL DE ABASTECIMENTO - CONAB. Acompanhamento da safra brasileira: Grãos - Safra 2018/19, Décimo Segundo Levantamento, https://www.conab.gov.br/infoagro/safras/graos/boletim-da-safra-de-graos. v. 6, n. 3, 127p, 2018.

[8] COMPANHIA NACIONAL DE ABASTECIMENTO - CONAB. Produção de grãos da safra 2020/21 segue como maior da história: 268,9 milhões de tonelada, 2020. https://www.conab.gov.br/ultimasnoticias/3691-producao-de-graos-da-safra-2020-21-segue-como-maior-da-historia-268-9-milhoes-detoneladas\#: : :text=De\%20acordo\%20com\%20o\%202\%C2\%BA,a\%20temporada\%20de\%202019\%2F2020.

[9] THIAGO, L.R.L.S., SILVA, J.M. Soja na alimentação de bovinos, In: Circular Técnica: Embrapa Gado de Corte, v.31, 6p., Campo Grande, 2005.

[10] ASSOCIAÇÃO BRASILEIRA DE NORMAS TÉCNICAS. NBR 14853, Madeira: determinação do material solúvel em etanol-tolueno e em diclorometano e em acetona, Rio de Janeiro, 2010b.

[11] ASSOCIAÇÃO BRASILEIRA DE NORMAS TÉCNICAS, NBR 7989, Pasta celulósica e madeira: determinação de lignina insolúvel em ácido, Rio de Janeiro, 2010a.

[12] ASSOCIAÇÃO BRASILEIRA DE NORMAS TÉCNICAS, NBR 13999, Papel, cartão, pastas celulósicas e madeira: determinação do resíduo (cinza) após a incineração a $525^{\circ} \mathrm{C}$, Rio de Janeiro, 2003b.

[13] AMERICAN SOCIETY FOR TESTING AND MATERIALS, ASTM D 790 - 07, Standard Test Methods for Flexural Properties of Unreinforced and Reinforced Plastics and Electrical Insulating Materials, 2007.

[14] AMERICAN SOCIETY FOR TESTING AND MATERIALS, ASTM D 638 - 03, Standard Test Method for Tensile Properties of Plastics, 2003.

[15] AMERICAN SOCIETY FOR TESTING AND MATERIAL, ASTM D 570, Standard Test Method for Water Absorption of Plastics, 1998.

[16] SILVA, M.S, NAVES, M.M.V., OLIVEIRA, R.B., et al., Composição química e valor protéico do resíduo de soja em relação ao grão de soja. Ciência e Tecnologia de Alimentos,v. 26, n.3, 2006.

[17] RAYMUNDO, E.A., RIBEIRO, R.B., CARVALHO, E.F., et al., "Análise de absorção de água em compósito de poliméro reforçado com fibras naturais", Cadernos UniFOA Edição Especial do Curso de Mestrado Profissional em Materiais, 2012.

[18] ENGLISH, B., FALK, R.H., "Factors that affect the application of wood fiberplastic composites Proceedings, Wood-Plastic Conference, Baltimore, p. 60-72, 2000.

[19] YAMAJI, F.M., BONDUELLE, A., "Utilização da serragem na produção de compósitos plásticomadeira", Revista Floresta, v. 34, n. 1, p. 59-66, 2004.

[20] MARTINS, E.H, VILELA, A.P., MENDES, R.F., et al. Soybean waste in particleboard production. Ciência e Agrotecnologia, v. 42, n. 2, p. 186-194, 2018.

[21] SHESHMANI, S., ASHORI, A., FARHANI, F. Effect of extractives on the performance properties of wood flour-polypropylene composites. Journal of Applied Polymer Science 123: 1563-1567, 2012.

[22] BHATIA, L., JOHRI, S., AHMAD, R. "An economic and ecological perspective of ethanol production from renewable agro waste", AMB Express, v. 2, n. 1, pp. 65, 2012.

[23] LOPEST.A., SCATOLINO, M., LOPES, T.A., et al., "Resistência a combustão e painéis MDP produzidos com resíduos da cultura da soja", III Congresso Brasileiro de Ciência e Tecnologia da Madeira, Florianópolis, Santa Catarina, Brasil, 2017.

[24] BARRICHELO, L., BRITO, J.O., "Usos diretos e propriedades da madeira para geração de energia”, Silvicultura, v. 2, n. 12, pp. 26-28, 1979.

[25] MIRANDA, M.R.S., "Bagaço de sorgo: estimativa de parâmetros cinéticos e pirólise analítica". Dissertação de M.Sc., Universidade Federal de Uberlândia, Uberlândia, MG, Brasil, 2011.

[26] SANTANA, J.A., SOUZA, N.G., CARDOSO, C.R. et al. Sodium, zinc and magnesium chlorides as additives for soybean hulls pyrolysis. J Therm Anal Calorim v. 125, pp. 471-481, 2016. 
[27] QING, Q., GUO, Q., ZHOU, L. et al. Comparison of alkaline and acid pretreatments for enzymatic hydrolysis of soybean hull and soybean straw to produce fermentable sugars. Industrial Crops Products, v. 109, pp. 391-397, 2017.

[28] CHINTA, S.K., KATKAR, P.M., JAFER, M.M. "Natural fibres reinforced gypsum composites," International Journal of Management Science and Engineering, v. 4, n. 3, pp. 318-325, 2013.

[29] POLETTO, M. Compósitos termoplásticos com madeira - uma breve revisão. Revista Interdisciplinar de Ciência Aplicada, v.2, n.4, 2017.

[30] TEIXEIRA, M.G., CÉSAR, S.F., "Produção de compósito com resíduo de madeira no contexto da ecologia industrial", Revista Madeira Arquitetura \& Engenharia, v. 7, n. 19, pp. 1-15, 2006.

[31] TITA, S.P.S., PAIVA, J.M.F., FROLLINI, E., "Resistência ao impacto e outras propriedades de compósitos lignocelulósicos: matrizes termofixas fenólicas reforçadas com fibras de bagaço de cana-de-açúcar", Polímeros: Ciência e Tecnologia, v. 12, n. 4, pp. 228-239, 2002.

[32] LEMOS, A.L., MARTINS, R.M., "Desenvolvimento e caracterização de compósitos poliméricos à base de poli (ácido lático) e fibras naturais", Polímeros: Ciência e Tecnologia, v.24, n. 2, pp. 190-197, 2014.

[33] PRACELLA, M., CHIONNA, D., ANGUILLESI, I., et al. Composites Science and Technology, v.66, n.13, pp.2218-2230, 2006.

[34] LI, X., TABIL, L.G., PANIGRAHI, S. Chemical Treatments of Natural Fiber for Use in Natural FiberReinforced Composites: A Review, Journal of Polymers and the Environment, v.15, n.1, pp.25-33, 2007.

[35] GUEDES, J.R., FLORENTINO, W.M., RODRIGUES, L.M., "Propriedades mecânicas de compósitos de polietileno de baixa densidade reforçado com fibra da palmeira real australina", $21^{\circ}$ Congresso Brasileiro de Engenharia e Ciência dos Materiais, Cuiabá, Mato Grosso, Brasil, 2014.

[36] STORK, R.R., ROCHA, M.C.G., "Compósitos de polietileno de baixa densidade e torta de mamona", $10^{\circ}$ Congresso Brasileiro de Polímeros, Foz do Iguaçu, Paraná, Brasil, Outubro, 2009.

[37] ROCHA, J.G., MULINAR, D.R., "Caracterização mecânica dos compósitos de PEBD reforçados com fibras da palmeira”, Cadernos UniFOA, v. 9, n. 1, pp.45-53, 2014.

[38] MOREIRA, T.M., SEO, E.M. Obtenção e caracterização de polímero reforçado com fibras da folha de milho. Matéria (Rio J.), v. 21, n. 4, p. 1054-1068, 2016.

[39] OLIVEIRA, et al., "Avaliação do uso de agente compatibilizante no comportamento mecânico dos compósitos PEBD reforçados com fibras de coco verde", Cadernos UniFOA, v. 5, n. 14, 2010.

[40] SANCHEZ, E.M.S., et al. "Compósito de resina de poliéster insaturado com bagaço de cana-de -açúcar: influência do tratamento das fibras nas propriedades”, Polímeros, v. 20, n.3, pp. 194-200, Out. 2010.

\section{ORCID}

Thalita Paula dos Santos

Hudson Venâncio Silva Garcia

Thaynara Andrade Lopes

Carolina Aparecida dos Santos

Ana Carolina Corrêa Furtini

Lourival Marin Mendes

José Benedito Guimarães Junior https://orcid.org/0000-0001-7682-8865

https://orcid.org/0000-0002-2609-7483

https://orcid.org/0000-0002-4825-2747

https://orcid.org/0000-0002-3469-9011

https://orcid.org/0000-0002-2106-6602

https://orcid.org/0000-0001-8713-405X

https://orcid.org/0000-0002-9066-1069 